\title{
$\mu$-Suppression during Action Observation and Execution Correlates with BOLD in Dorsal Premotor, Inferior Parietal, and SI Cortices
}

\author{
Dan Arnstein, ${ }^{1}$ Fang Cui, ${ }^{1,3}$ Christian Keysers, ${ }^{1,3}$ Natasha M. Maurits, ${ }^{2}$ and Valeria Gazzola ${ }^{1,3}$ \\ Departments of ${ }^{1}$ Neuroscience and ${ }^{2}$ Neurology, University Medical Center Groningen, University of Groningen, 9713 GZ Groningen, The Netherlands, and \\ ${ }^{3}$ Netherlands Institute for Neuroscience, Royal Netherlands Academy for Arts and Sciences, 1105BA Amsterdam, The Netherlands
}

The discovery of mirror neurons in the monkey, that fire during both the execution and the observation of the same action, sparked great interest in studying the human equivalent. For over a decade, both functional magnetic resonance imaging (fMRI) and electroencephalography (EEG) have been used to quantify activity in the human mirror neuron system (MNS) - yet, little is still known about how fMRI and EEG measures of the MNS relate to each other. To test the frequent assumption that regions of the MNS as evidenced by fMRI are the origin of the suppression of the EEG $\mu$-rhythm during both action execution and observation, we recorded EEG and BOLD-fMRI signals simultaneously while participants observed and executed actions. We found that the suppression of the $\mu$-rhythm in EEG covaried with BOLD activity in typical MNS regions, inferior parietal lobe (IPL), dorsal premotor (dPM) and primary somatosensory cortex (BA2), during both action observation and execution. In contrast, in BA44, only nonoverlapping voxels correlated with $\mu$-suppression during observation and execution. These findings provide direct support for the notion that $\mu$-suppression is a valid indicator of MNS activity in BA2, IPL, and DPM, but argues against the idea that mirror neurons in BA44 are the prime source of $\mu$-suppression. These results shed light on the neural basis of $\mu$-suppression and provide a basis for integrating more closely the flourishing but often separate literatures on the MNS using fMRI and EEG.

\section{Introduction}

The discovery of mirror neurons (Gallese et al., 1996; Umiltà et al., 2001; Kohler et al., 2002) has sparked great interest in measuring the activity of the mirror neuron system (MNS) in humans (Keysers, 2009), and psychiatric patients, in particular (Iacoboni and Dapretto, 2006; Rizzolatti et al., 2009). Two methods are dominant: functional magnetic resonance imaging (fMRI) and electroencephalography (EEG). Although they are assumed to both measure activity in the same MNS, little is known about how these measures of the MNS relate to each other.

fMRI typically maps the MNS as voxels active when a participant executes and perceives similar actions (Grèzes et al., 2003; Dinstein et al., 2007; Gazzola and Keysers, 2009; Turella et al.,

Received Feb. 22, 2011; revised July 7, 2011; accepted July 19, 2011.

Author contributions: C.K. and V.G. designed research; D.A., F.C., N.M.M., and V.G. performed research; C.K. contributed unpublished reagents/analytic tools; D.A., F.C., C.K., N.M.M., and V.G. analyzed data; D.A., F.C., C.K., N.M.M., and V.G. wrote the paper.

The work was supported by a Marie Curie Excellence Grant of the European Commission and a VIDI grant of the Dutch Science Foundation to CK [Netherlands Organisation for Scientific Research (NWO), ] VENI grant of the NWO to V.G., a Fulbright scholarship to D.A., and the China Scholarship Council for State Scholarship Fund to F.C. We thank Alessandro Rigon, Paolo Toffanin, Nikola Valchev, and Idil Kokal for their help in preparing the experiment.

The authors declare that they have no competing financial interests for this study.

D.A. and F.C. contributed equally, and C.K., N.M.M., and V.G. contributed equally.

This article is freely available online through the J Neurosci Open Choice option.

Correspondence should be addressed to Valeria Gazzola, Netherlands Institute for Neuroscience, and Royal Neth-

erlands Academy for Arts and Sciences, Amsterdam, Meibergdreef 47, 1105BA Amsterdam, The Netherlands. E-mail: v.gazzola@nin.knaw.nl.

DOI:10.1523/JNEUROSCI.0963-11.2011

Copyright $\odot 2011$ the authors $\quad 0270-6474 / 11 / 3114243-07 \$ 15.00 / 0$ 2009)—so-called shared voxels (sVx) (Gazzola and Keysers, 2009). Regions involved in hand-action execution are localized using relatively complex movements (reaching and manipulating an object). EEG typically quantifies MNS activity using the modulations of an $\sim 10 \mathrm{~Hz}$ rhythm (lower $\mu$ ) recorded over the sensorimotor cortex. $\mathrm{Mu}$ is measured while participants observe hand actions (Gastaut, 1952; Pineda et al., 2000; Oberman et al., 2005; Pineda, 2005; Southgate et al., 2009), and while repeatedly executing the same action (Pineda et al., 2000; Oberman et al., 2005; Raymaekers et al., 2009), e.g., closing and opening a hand. $\mathrm{Mu}$ power is reduced during both action observation and execution. $\mathrm{Mu}$ is most powerful in the primary somatosensory cortex (SI) (Salmelin et al., 1995; Ohara et al., 2000; Caetano et al., 2007); however, we lack evidence for the assumption that regions of the MNS, the ventral premotor cortex in particular (Pineda, 2005), are responsible for $\mu$-modulation and that fMRI and $\mu$-suppression experiments measure the functioning of the same MNS. Elegant MEG experiments also investigated an $\sim 20 \mathrm{~Hz}$ $\mu$-component which rebounds after action observation and execution (Hari et al., 1998), but because optimal designs to study 10 $\mathrm{Hz}$ and $20 \mathrm{~Hz}$ components differ, we focus here on the most studied, $10 \mathrm{~Hz}$ component, and will use $\mu$ as shorthand for that component alone.

Hence, we simultaneously measured EEG and fMRI while participants observed and executed hand actions. If fMRI and $\mu$-suppression EEG both record the activity of the same underlying MNS, on a trial-by-trial basis, the $\mu$-power should be negatively correlated with the BOLD signal during both observation 


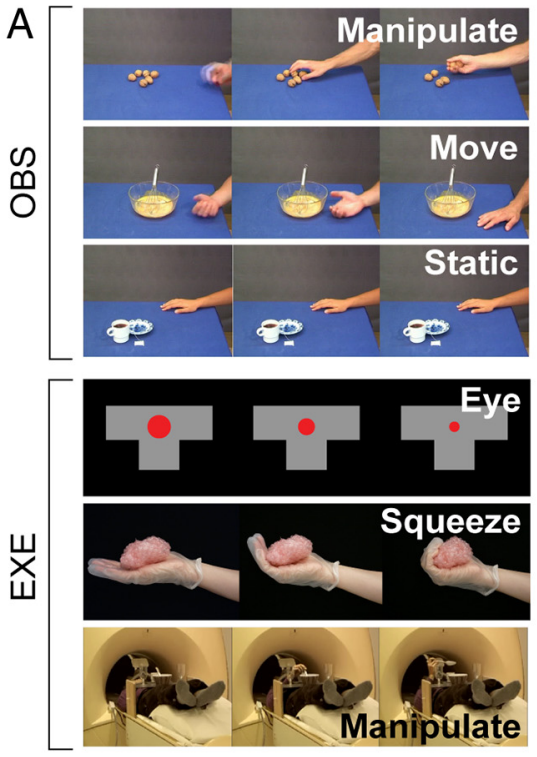

C
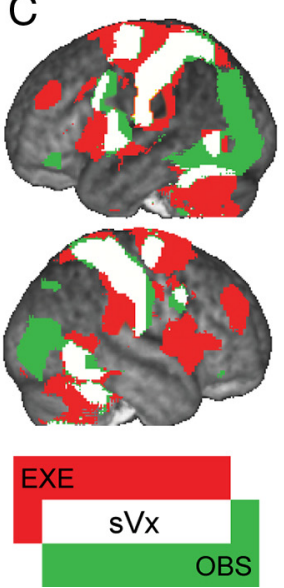

D
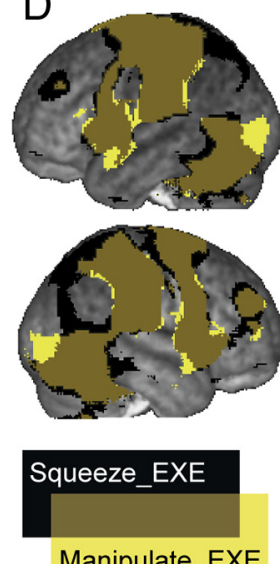

B

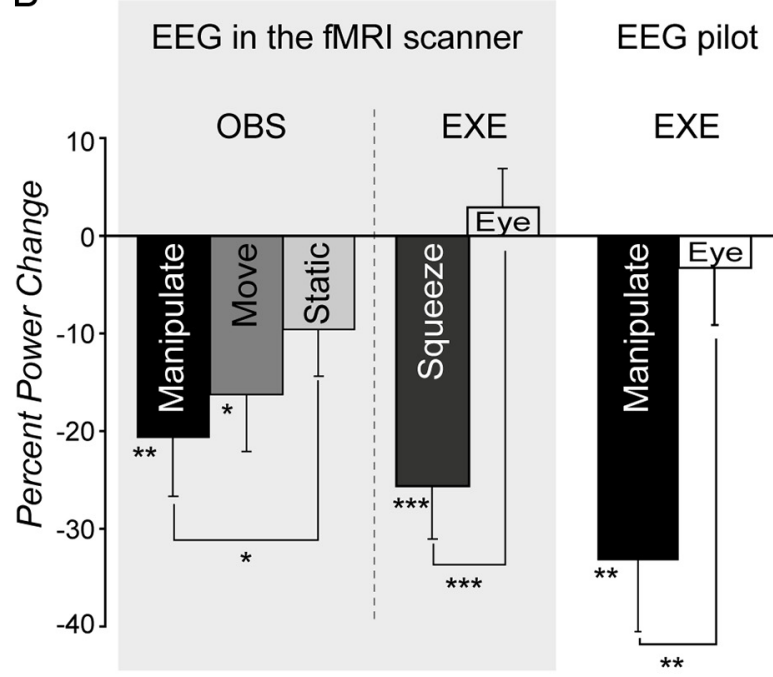

$\mathrm{E}$

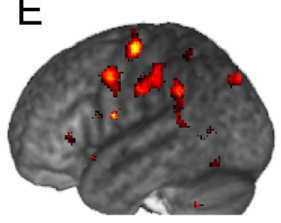

F

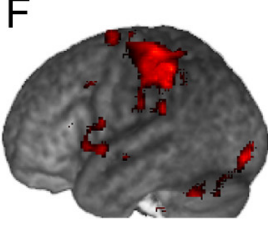

G
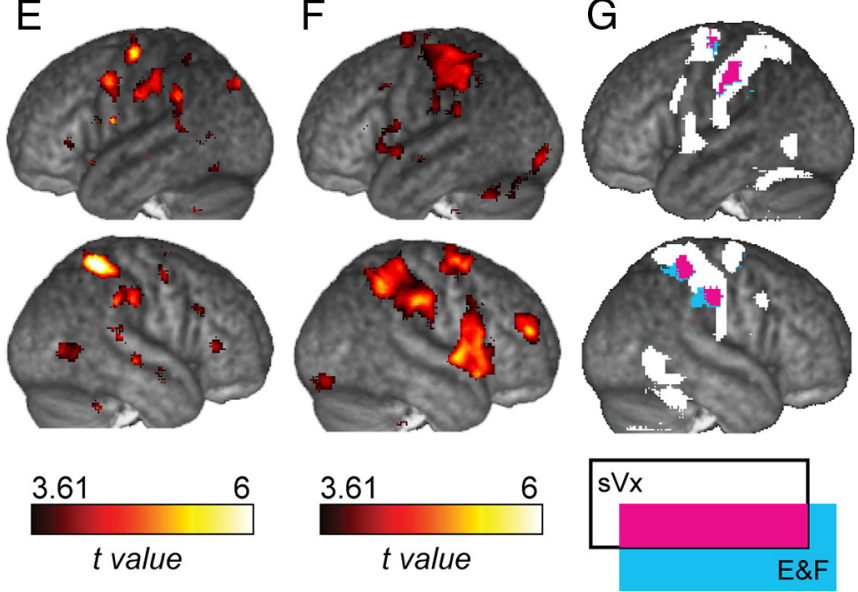

Figure 1. A, Snapshots from the different experimental conditions. $\boldsymbol{B}$, Percentage $\mu$-power change relative to average baseline power in $C 3$ while participants observed or performed actions within the scanner environment and while participants performed the Manipulate_EXE condition in the pilot experiment. ${ }^{*} p<0.05,{ }^{* *} p<0.01,{ }^{* * *} p<0.001$. t tests against zero ( $=$ baseline) when over a bar or matched-sample $t$ test when over square brackets. C, SVX fMRI localizer, i.e., voxels activated during action execution (Manipulate_EXE-Eye_EXE, punc $<0.001$, red), during action observation (Manipulate_OBS-Move_OBS, punc $<0.01$ and Manipulate_OBS-Static_OBS, punc $<0.01$, green) or both (white, SVx). D, Comparison of voxels significantly activated during Squeeze_ EXE (black) and Manipulate_EXE (yellow). E, Voxels with $\mu$-power correlating negatively with BOLD signal during OBS ( $p_{\text {unc }}<0.001$, all clusters survive $\left.p_{\text {FDR }}<0.05\right)$. F, Same for Squeeze_EXE. $\bar{G}$, sVx (white as in C), voxels correlating with $\mu$-power suppression during observation and execution (i.e., overlap of $\boldsymbol{E}$ and $\boldsymbol{F}$, blue), and their overlap (pink).

and execution of actions, mainly in regions typically associated with the human MNS. These include Brodmann area (BA) 44 and the inferior parietal lobe (IPL), and other regions that have been associated with the MNS more recently (Keysers and Gazzola, 2009), including the supplementary motor area (SMA) (Mukamel et al., 2010; Gazzola and Keysers, 2009), the dorsal premotor cortex (dPM) (Cisek and Kalaska, 2004; Gazzola and Keysers, 2009) and BA2 (Gazzola and Keysers, 2009; Keysers et al., 2010).

\section{Materials and Methods \\ Participants}

Nineteen subjects (11 male, average age 21.6 years, range: $18-28$ years) participated in the experiment. Prescreening excluded subjects with a history of neurological disorders, impaired vision after correction, or who were left-handed. The research was approved by the Medical Ethical Commission of the University Medical Center Groningen (Netherlands) and all subjects provided informed consent.

\section{Experimental task}

The experiment consisted of three sessions, one of observation and two of execution.
Observation session (OBS)

Participants viewed one of three types of movies (Fig. $1 A$ ): a right hand entering from the right side of the screen to manipulate an object on a table (e.g., watering a plant or cracking nuts; Manipulate_OBS); a right hand entering the screen and moving continuously without interacting with the objects on the table (Move_OBS); or a right hand resting on the table close to the object (Static_OBS). The hand and the object in the Static_OBS condition were positioned not to imply a goal directed action. To enable a correlation analysis between $\mu$-power and BOLD, we chose these three conditions to vary in how strongly they activate the MNS, with Manipulate_OBS, Move_OBS and Static_OBS expected to activate the MNS strongly, mildly and weakly.

The duration of the movies, either 2 or $3 \mathrm{~s}$, was chosen based on the optimal time needed to manipulate the object. The two control conditions were then matched for duration. To avoid habituation, each movie was shown only once in the experiment, and each object was used exactly for one movie of each condition. Additionally, 4 different actors played in the movies (balanced across conditions) to vary the hand and kinematics across movies. The background was kept constant and consisted of a table covered with a blue tablecloth in front of a wall covered by a grayish fabric.

Each condition was presented in a block design. Each block lasted $7 \mathrm{~s}$ and included a random set of two $2 \mathrm{~s}$ stimuli and one $3 \mathrm{~s}$ stimulus of the 
same condition. Blocks were presented in a pseudo-randomized order (no more than 2 consecutive repetitions of the same condition were allowed) that differed for each subject. There were 13 blocks (i.e., repetitions) for each condition separated by a random 10-14 s interval in which a gray and blue rectangle, that resembled the colors of the table and the wall, were presented together with a skin-colored fixation cross in the middle. This baseline was chosen to limit the "surprise transition effect" between the baseline and the beginning of each block.

Movies were recorded using a digital video camera (Sony DSRPDX10P) and elaborated using Adobe Premiere (www.adobe.com) and Windows MovieMaker (www.microsoft.com).

\section{Manipulate versus eye execution session (Manipulate_EXE vs Eye_EXE)}

In the scanner, a T-shaped plastic table was placed over the waist of the participant. A coffee cup was placed at the end closest to the participant, a wine glass was placed on the right end, and a bowl and spoon were placed at the intersection (Fig. 1A). Subjects had to watch a screen on which a circle appeared on the bottom, right, or intersection of a diagram of the table. If the circle was green, participants had to manipulate the corresponding object on the real table with their hand (Manipulate_ EXE) by grasping the cup and bringing it toward their mouth, grasping the wine glass and swirling it, or grasping the spoon and manipulating it as if to ladle soup. The circle would shrink, and subjects were instructed to perform the action until the circle disappeared. Every block consisted of all three actions in a random order, with the circles timed to make two of the actions last $3 \mathrm{~s}$ and one lasting $4 \mathrm{~s}$. Participants were prevented from seeing themselves perform the actions, and were trained in the task outside the scanner and again in the scanner immediately before the beginning of the session. If the circle was red (Eye_EXE), participants had to move their eye gaze to the location of the circle on the screen instead of moving their hands. Except for the color of the circles, the visual stimuli and their timing were matched in the Eye_EXE and Manipulate_EXE conditions. Block order was pseudo-randomized (no more than 2 consecutive trials of the same condition) both within and between subjects. Participants completed 13 blocks for each condition. Blocks were separated by a random 10-14 s interval showing a diagram of the table with a small gray circle in the middle as fixation point. During this time, participants were requested to rest their hand in a comfortable position close to the table and to avoid contact with the objects.

These two motor conditions were chosen to resemble those typically used in fMRI experiments (12-17).

\section{Squeeze execution session (Squeeze_EXE)}

Throughout the session, participants wore a glove on the right hand, with bubble-wrap packing material attached to the palm (Fig. $1 \mathrm{~A}$ ). During each of the 20 blocks, participants were shown a sequence of four green circles of decreasing size, each lasting $1 \mathrm{~s}$. At the onset of each green circle, participants were instructed to squeeze the material gently between the fingers and palm, leading to 4 squeezes in each block. Blocks were separated by a random 10-14 s interval with a gray circle in the middle of the screen. Participants were prevented from seeing themselves perform the actions, and were trained in the task outside the scanner and again in the scanner immediately before the beginning of the session. This run was performed to resemble the motor task of typical EEG experiments $(23,27,30)$.

The observation session was always completed before the execution sessions to avoid motor priming. The experimental tasks were designed to induce modulations in $\mu$ power as well as the BOLD signal in the MNS. All stimuli were delivered using Presentation (www.neuro-bs.com), and projected with an LCD projector on a semi-opaque screen placed at the head end of the bore and seen through a mirror placed on the head coil.

\section{Data acquisition \\ $f M R I$}

A Philips Intera 3T Quaser whole-body scanner equipped with a circular sense head coil was used. We used a T2*-weighted echoplanar sequence with 39 interleaved $3.5 \mathrm{~mm}$ thick axial slices with no gap for functional imaging $\left(\mathrm{TR}=2000 \mathrm{~ms}, \mathrm{TE}=30 \mathrm{~ms}\right.$, flip angle $=80^{\circ}, \mathrm{FOV}=224 \mathrm{~mm} \times$
$224 \mathrm{~mm}, 64 \times 64$ matrix of $3.5 \mathrm{~mm}$ isotropic voxels). The slice acquisition frequency $(19.5 \mathrm{~Hz})$ was selected to minimize noise in the $\mu$ frequency band $(8-13 \mathrm{~Hz})$. At the end of the functional scanning, a T1-weighted anatomical image $(1 \times 1 \times 1 \mathrm{~mm})$, parallel to the bicommissural plane and covering the whole brain, was acquired.

\section{EEG}

An MR-compatible 32-channel BrainAmp system (Brain Products, Munich, Germany) was used. The 29 scalp electrodes were set up according to the international 10-20 system. One additional channel was dedicated to EOG and two channels to EKG. The reference electrode was positioned at $\mathrm{FCz}$ (between $\mathrm{Fz}$ and $\mathrm{Cz}$ ). The impedances of all channels were maintained below $20 \mathrm{k} \Omega$. All data were recorded using the BrainVision Recorder 1.03 software (Brain Products) with a sampling frequency of 5 $\mathrm{kHz}$.

\section{Data processing}

\section{fMRI}

fMRI data were preprocessed using SPM8 (www.fil.ion.ucl.ac.uk). All echoplanar images (EPIs) were slice-time corrected and realigned to the subject's mean EPI. $T_{1}$ images were then co-registered to the mean EPI, segmented, and the gray matter was used to estimate the normalization parameters which were then applied to all EPIs. Normalized EPIs were smoothed with a $9 \mathrm{~mm}$ isotropic FWHM Gaussian kernel.

For most subjects, there was a drop in BOLD signal intensity over the left parietal lobe, likely an artifact caused by the cables connecting the EEG electrodes to the amplifier, which were often pressed close to the subject's scalp by the MRI head coil. These reduced EPI signals lead SPM8 to consider these voxels out of the brain. To solve this problem: (a) all 19 subjects' smoothed mean EPIs were averaged into a grand mean EPI; (b) this grand mean EPI was divided by each subject's smoothed mean EPI; (c) we then multiplied, for each subject separately, all the smoothed EPIs by the subject's correction map obtained in point (b). This procedure allowed SPM8 to accurately identify the boundaries of the left parietal lobe for all subjects. Because the same correction factor was applied to all EPI images of the same participant, this procedure does not bias the General Linear Model that examines changes over time.

\section{EEG}

BrainVison Analyzer 1.05 software (Brain Products, Munich, Germany) was used for off-line correction of MRI scanner artifacts and pulse artifacts as described previously (Allen et al., 1998, 2000). The data were then filtered with a $40 \mathrm{~Hz}$ low-pass filter ( independent component analysis was performed using the TDsep (Ziehe et al., 2000) algorithm (http://sccn.ucsd.edu/eeglab/) and components visually identified as ocular artifacts or residual MRI artifacts were removed.

According to the literature, EEG $\mu$-suppression is clearest in the contralateral hemisphere (Harmon-Jones, 2006; Perry and Bentin, 2009). Since our subjects used the right hand in the Manipulate_EXE and only observed right hands in the Manipulate_OBS condition, we mainly focus on $\mu$-power recorded from C3. The data from C3 were therefore convolved with a $10 \mathrm{~Hz}$ Morlet wavelet (Morlet parameter =5) in BrainVision Analyzer to obtain a time course of $\mu$-power throughout the experiment.

To minimize the impact of remaining artifacts, $\mu$-power values differing more than two SDs from the mean were rejected and replaced using a linear interpolation based on the previous and subsequent power values. Approximately $2.5 \%$ of the data were replaced using this method.

Additionally, we observed that when scanner and/or pulse artifacts survived the correction procedures, the correction failed on all sites, not just C3. Therefore, we computed correlations between alpha power at C3 and every other site for each block and baseline, and when the average correlation was $>0.8$, the block or baseline was labeled as contaminated. Visual inspection suggested that 0.8 was an effective threshold for eliminating blocks with residual artifacts (61/874) while sparing clean blocks. In the combined EEG/fMRI analyses described below, bad baselines (56/874) were excluded from the calculation of average 
baseline power, and bad blocks were modeled separately with a boxcar predictor of no interest.

\section{Data analyses \\ fMRI task effect and sVx identification}

At the first, subject level, a general linear model (GLM) was applied to the fMRI data, separately for each session. Each condition within each session was modeled by using a standard boxcar function convolved with the hemodynamic response function (HRF). Six additional predictors of no interest were entered in the GLM to account for translations and rotations of the head. For all analyses, the high-pass filter was chosen such that the period was equal to the maximum time between repetitions of a condition plus $15 \mathrm{~s}$ (to account for hemodynamic delays) plus $10 \%$. Blocks labeled as bad (due to high intercorrelations between EEG sites or poor performance of the task) were modeled separately with a boxcar predictor of no interest. Data were then analyzed at the second level using $t$ tests on the parameter estimates for each subject obtained at the first level.

Shared voxels ( $\mathrm{s} V \mathrm{x}$; i.e., voxels activated during both action execution and observation) were defined at the second level as in (Gazzola and Keysers, $2009)$ using the conjunction of contrast Manipulate_EXE $>$ Eye_EXE $(p<0.001)$, Manipulate_OBS $>$ Move_OBS $(p<0.01)$ and Manipulate OBS $>$ Static_OBS $(p<0.01)$. For Manipulate_OBS $>$ Move_OBS and Manipulate_OBS $>$ Static_OBS, an uncorrected $p<0.01(k>10)$ threshold was used because the conjunction of the two contrasts has a false-positive rate of between 0.01 and .0001 , approximating a $p<0.001$ threshold (Gazzola and Keysers, 2009).

Given the difference in motor task used by typical fMRI and EEG experiments, we also examined the impact of using Squeeze_EXE instead of Manipulate_EXE to define the sVx by correlating the second-level $t$ maps of Squeeze_EXE and Manipulate_Exe and by overlapping the significantly activated voxels.

\section{EEG-only analyses}

Before running the combined EEG-fMRI recordings, we investigated whether the more complex task commonly used in fMRI experiments would also produce measurable $\mu$-suppression. With this aim we conducted a pilot EEG experiment outside the scanner in which 13 student participants (none of which participated in the main study) performed the Manipulate_EXE and Eye_EXE task. Figure $1 B$ shows that, compared with average baseline power, Manipulate_EXE did produce $\mu$-suppression, and more so than Eye_EXE.

Unfortunately, during the combined EEG-fMRI recordings, the EEG data corresponding to the Manipulate_EXE condition were contaminated by large artifacts that could not be corrected because their shape varied from trial to trial. Since these artifacts were frequent only in this condition, we suspect that the arm movements required for this task caused head movements, and therefore EEG sensor movements, that, although small $(<1.7 \mathrm{~mm}$ based on MRI realignment parameters), caused irregular magnetic induction artifacts that could not be removed. In the main analyses we therefore only analyzed the Squeeze_EXE and Eye_EXE conditions which did not show this type of artifact.

For the observation, Squeeze_EXE, Eye_EXE conditions collected during the EEG-fMRI recordings, and for the Manipulate_EXE and Eye_ EXE in the pilot study, the $\mu$-power was averaged during the relevant blocks and expressed as a percentage of power change relative to the average power during the baseline of each run. The baseline power was calculated based on the epoch 7 to $1 \mathrm{~s}$ before a block would start rather than over the full interblock interval. This choice of baseline allows at least $3 \mathrm{~s}$ for the $\mu$-rebound to occur without affecting the baseline estimate, and we chose to terminate the baseline $1 \mathrm{~s}$ before the block onset because a $10 \mathrm{~Hz}$ wavelet defined with a Morlet parameter of 5 extends close to $1 \mathrm{~s}$ in either direction.

\section{Combined EEG/fMRI analyses}

Observation. To focus our analysis on the differences in $\mu$ power between the three conditions (Manipulate_OBS, Move_OBS and Static_OBS) rather than the differences between the task and the baseline (which extensive low-level visual activations could account for), we removed the
BOLD variance which was common to all the conditions. Specifically, we defined a GLM at the first, subject level that contained a single boxcar predictor containing all periods of visual stimulation and an orthogonalized C3-power predictor. The C3 $\mu$-predictor was set to zero during baselines and to the actual instantaneous $\mu$-power minus the average baseline power during Manipulate_OBS, Move_OBS, and Static_OBS blocks, then orthogonalized with respect to the visual boxcar task predictor. The $\mu$-predictor and boxcar visual predictor were then convolved with the HRF, and the convolved $\mu$-predictor was subsampled at $0.5 \mathrm{~Hz}$ (at the time of acquisition of the reference slice of each fMRI volume) and standardized to zero mean and unit variance.

When building the $\mu$-predictors, the baseline power was calculated as described in EEG-only analyses.

Squeeze_EXE. For the combined EEG/fMRI analysis of Squeeze_EXE, a GLM was applied with C3 $\mu$-power as a predictor. The $\mu$-predictor was set to the actual $\mu$-power minus the average baseline power (calculated between 7 and $1 \mathrm{~s}$ preblock) during Squeeze_EXE condition and to zero at all other times. The predictor was then convolved with the HRF, subsampled and standardized.

Unless otherwise specified, all results are presented at a threshold of $p<0.001$ uncorrected and all clusters also survive to FDR-corrected $p<$ $0.05(k>10)$.

\section{Results}

\section{EEG}

During observation, $\mu$-suppression was significant at C3 while observing actors manipulate objects and actors move their hands but not while viewing static images of the hands and objects (Fig. $1 B)$. A three-condition ANOVA revealed a significant effect of condition $\left(F_{(2,36)}=3.6, p<0.05\right)$, and post hoc testing (LSD) showed this was due to Manipulate_OBS eliciting greater $\mu$-suppression than Static_OBS.

Relative to average baseline power, there was also significant $\mu$-suppression at C3 while participants squeezed an object (bubble foil, Squeeze_EXE; $t=-4.680, p<0.001$ ), replicating the finding of typical EEG $\mu$-suppression studies. This $\mu$-suppression also exceeded that during Eye_EXE $(t=-4.820, p<0.001$, Fig. $1 B)$, suggesting that $\mu$-suppresion during Squeeze_EXE was not due to unspecific visual or executive processes.

\section{fMRI}

In line with our previous fMRI experiments (Gazzola et al., 2006, 2007; Gazzola and Keysers, 2009), we localize the MNS by mapping $s \mathrm{Vx}$, i.e., voxels with BOLD activity larger while participants reach for and manipulate objects than while performing eye movements (Manipulate_EXE-Eye_EXE, $p<0.001$ ) AND larger while viewing actors manipulate objects than both while viewing them move their hand without manipulating an object and while viewing a still image of the hand and object (Manipulate_OBSMove_OBS, $p<0.01$ and Manipulate_OBS-Static_OBS, $p<$ $0.01)$. The locations of the $\mathrm{sVx}$ are consistent with past findings (Grèzes et al., 2003; Dinstein et al., 2007; Filimon et al., 2007; Gazzola et al., 2006, 2007; Gazzola and Keysers, 2009; Turella et al., 2009; Caspers et al., 2010) and include BA2, BA44, dPM, the SMA, IPL and SPL (Fig. 1C).

To compare the motor properties of the MNS defined in typical fMRI and EEG experiments, we compared the pattern of BOLD activation induced by Manipulate_EXE and Squeeze_ EXE. Computing a spatial correlation between the $t$-maps obtained at the second level of analysis of the BOLD signal for Squeeze_EXE and Manipulate_EXE revealed a high correlation $(r=0.83)$ and Squeeze_EXE activated a network of brain regions very similar to, although slightly smaller than, Manipulate_EXE (Fig. 1D). 
Table 1. Overlap between the MNS based on EEG and fMRI criteria

\begin{tabular}{|c|c|c|c|c|c|c|c|}
\hline \multirow{2}{*}{$\begin{array}{l}\text { Cluster size (voxels) } \\
216\end{array}$} & \multicolumn{3}{|c|}{ MNI coordinates $x, y, z(\mathrm{~mm})$} & \multirow{2}{*}{$\begin{array}{l}T \\
4.65\end{array}$} & \multirow{2}{*}{$\begin{array}{l}\text { Hemisphere } \\
\mathrm{L}\end{array}$} & \multirow{2}{*}{$\begin{array}{l}\text { Region } \\
\text { Postcentral gyrus }\end{array}$} & \multirow{2}{*}{$\begin{array}{l}\text { Cytoarchitectonic area } \\
\text { Area } 2\end{array}$} \\
\hline & -50 & -30 & 45 & & & & \\
\hline & -60 & -20 & 32 & 4.61 & $\mathrm{~L}$ & Supramarginal gyrus & $\mathrm{IPC}(\mathrm{PFt})$ \\
\hline & -54 & -28 & 43 & 4.46 & $\mathrm{~L}$ & Inferior parietal lobule & IPC (PFt) \\
\hline & -62 & -22 & 36 & 4.42 & L & Supramarginal gyrus & $\mathrm{IPC}(\mathrm{PFt})$ \\
\hline 130 & 54 & -24 & 34 & 4.87 & $\mathrm{R}$ & Supramarginal gyrus & IPC (PFt) \\
\hline 127 & 38 & -46 & 58 & 6.61 & $\mathrm{R}$ & Superior parietal lobule & SPL (7PC) \\
\hline 25 & -36 & -14 & 62 & 5.57 & $\mathrm{~L}$ & Precentral gyrus & Area 6 \\
\hline
\end{tabular}

Voxels with sVx properties and BOLD signal significantly negatively correlated with $\mu$-power during action observation and execution (pink clusters of Fig. 1G)." $T$ " refers to the peak correlation between $\mu$-power and BOLD signal during $0 \mathrm{BS}$; "Region" to a macroanatomical description of the location of the peak; and "Cytoarchitectonic area" to the label the anatomy toolbox associates to the peak (if available). L, Left; R, right; IPC, inferior parietal cortex; SPL, superior parietal lobe.

\section{Combined EEG/fMRI}

Figure 1, $E$ and $F$, shows the voxels in which the BOLD signal was negatively correlated with $\mu$-power (i.e., higher BOLD activity in trials with higher $\mu$-suppression) while participants observed (Fig. $1 E$ ) or executed (Squeeze_EXE, Fig. $1 F$ ) actions. In accordance with our predictions, during observation $\mu$-suppression covaried with BOLD signal in regions typically associated with the MNS: BA2, BA44, dPM, the SMA, and IPL. The same was true during action execution. Furthermore, many of the voxels that correlated with $\mu$-suppression during Squeeze_EXE also correlated with $\mu$-suppression during observation (Fig. $1 G$, in blue). This was true in left BA2, left dPM, bilateral IPL and right SPL. However, voxels in BA44 correlating with $\mu$-suppression were different during action observation and action execution.

Furthermore, we found that all four clusters showing a correlation between BOLD signal and $\mu$-suppression overlapped with sVx (Fig. 1G, in pink; Table 1). This was true although the motor component of sVx was defined using Manipulate_EXE-Eye_EXE while the correlates of motor $\mu$-suppression were localized using Squeeze_EXE.

\section{Discussion}

It has been assumed (1) that the MNS in general, and BA44 and the IPL in particular, are responsible for modulating $\mu$-power during action execution and observation (Oberman et al., 2005; Pineda, 2005) and (2) that experiments using fMRI and $\mu$-suppression to study the function of the MNS in clinical populations, autism spectrum disorders in particular, study the integrity of the same system (Iacoboni and Dapretto, 2006). Here we tested these assumptions by simultaneously recording EEG and fMRI of our participants during both action execution and observation.

Correlating $\mu$-suppression with the BOLD signal revealed that, in a number of brain regions, BOLD activity covaried with $\mu$-suppression in the EEG during the squeezing of a bubble foil and the same was true during the observation of stimuli varying in how strongly they should activate the MNS (static images, hand movements, and hand-object manipulations). In both cases, these regions almost exclusively included regions that have been associated with the MNS in the literature: BA44, IPL, dPM and BA2 (Kohler et al., 2002; Grèzes et al., 2003; Keysers et al., 2003, 2010; Cisek and Kalaska, 2004; Raos et al., 2004; Gazzola et al., 2006, 2007; Iacoboni and Dapretto, 2006; Dinstein et al., 2007; Filimon et al., 2007; Rozzi et al., 2008; Evangeliou et al., 2009; Gazzola and Keysers, 2009; Keysers, 2009; Keysers and Gazzola, 2009; Kilner et al., 2009; Turella et al., 2009; Caspers et al., 2010; Rizzolatti and Sinigaglia, 2010). Overlapping these two separate analyses revealed that three of these regions, IPL, dPM and BA2, contained voxels of which the BOLD signal correlated with the amount of $\mu$-suppression measured in the EEG during both the observation conditions and while participants squeezed the bubble foil. Importantly, these clusters of voxels with BOLD signal correlating with $\mu$-suppression during both execution and observation also overlapped with the mirror neuron system of our participants defined as typical fMRI experiments would.

The IPL has always been considered one of the two core regions of the MNS: mirror neurons have been recorded in this region in the monkey (Rozzi et al., 2008) and human fMRI experiments have consistently shown that this region is active during both action observation and execution (Grèzes et al., 2003; Gazzola et al., 2006, 2007; Filimon et al., 2007; Gazzola and Keysers, 2009; Turella et al., 2009; Caspers et al., 2010).

The dPM has been less intensively investigated in the monkey for the presence of mirror neurons, but it contains mirror-like neurons active both while moving a cursor on a screen and while witnessing the cursor being moved by another individual (Cisek and Kalaska, 2004). In humans, the dPM is also very consistently activated both during action observation and execution (Grèzes et al., 2003; Gazzola et al., 2006, 2007; Filimon et al., 2007; Gazzola and Keysers, 2009; Turella et al., 2009; Caspers et al., 2010), and is therefore increasingly incorporated into models of the MNS (Keysers and Gazzola, 2009).

BA2 finally has not been investigated at all for the presence of mirror neurons using single cell recordings in monkeys, but ${ }^{14} \mathrm{C}$ deoxyglucose studies have shown that this region has enhanced metabolism during action observation and execution (Raos et al., 2004; Evangeliou et al., 2009). In humans this region is consistently activated both during the observation and the perception (observation and listening) of hand actions (Grèzes et al., 2003; Gazzola et al., 2006, 2007; Dinstein et al., 2007; Filimon et al., 2007; Gazzola and Keysers, 2009; Turella et al., 2009; Caspers et al., 2010). The pattern of activity in this region provides information about which of two actions is being performed during both action perception and execution (Etzel et al., 2008) and voxels with coordinates in BA2 show repetition suppression both during action observation and execution (Dinstein et al., 2007). Because this region represents the highest level of proprioceptive processing in SI and receives input from regions containing mirror neurons in the monkey, it has been proposed that BA2 activity during action observation may represent a somatosensory simulation of what observed movements would feel like, and this somatosensory simulation is thought to integrate and supplement the motor simulation performed in premotor regions (Keysers et al., 2010). Interestingly, BA2 not only seems to have mirror properties-it is also part of the sensorimotor strip in which $\mu$-power is strongest (Salmelin and Hari, 1994; Salmelin et al., 1995; Ohara et al., 2000; Caetano et al., 2007), and might therefore be particularly suited for modulating $\mu$-power during action observation and execution.

Interestingly, the fourth region, BA44, which has often been considered the most likely source of $\mu$-suppression during action observation (Pineda, 2005) contained voxels that predicted $\mu$-suppression during observation trials and other voxels that 
predicted $\mu$-suppression during action execution trials, but none that predicted both, arguing against the idea that mirror neurons in this region would be the prime source of modulation of $\mu$-power.

A caveat of our correlational approach is that although a brain region causing $\mu$-suppression would be expected to have a BOLD signal that correlates with $\mu$-suppression during action observation and execution, if a number of regions show such correlation, it might be that only one directly causes $\mu$-suppression while the others show such correlation by virtue of their functional connectivity or shared input with that region. In our case, this would suggest that some, but maybe not all, of the regions including the BA2, IPL, SPL or dPM cause $\mu$-suppression during action observation and execution but that BA44 is less likely to do so. Repeating the experiment with a high density EEG system might help localize the origin of $\mu$-suppression. Additionally, fMRI is currently acquired at a rate of a slice every $\sim 50 \mathrm{~ms}$. This generates artifacts with a basic frequency of $20 \mathrm{~Hz}$, making it difficult to study $\mu$-suppression in the beta range ( $\sim 20 \mathrm{~Hz}$, Hari et al., 1998). As faster acquisition methods develop, the frequency of these artifacts will increase, and the beta range will become amenable to an analysis similar to the one we have performed for the alpha range.

Additionally, the overlap between $\mathrm{sVx}$ and voxels correlating with $\mu$-suppression provides evidence that fMRI experiments identifying brain regions involved both during action observation and action execution $(\mathrm{sVx})$ and EEG experiments measuring $\mu$-suppression indeed quantify the activity of overlapping neural substrates. This was true despite defining the regions correlating with $\mu$-suppression using the Squeeze_EXE condition in line with previous EEG experiments while defining the $s \mathrm{Vx}$ using a different and more complex condition, Manipulate_EXE, in line with previous fMRI experiments. This overlap provides an empirical basis for combining evidence from EEG and fMRI experiments to study the integrity of the MNS in clinical populations, autism in particular (Iacoboni and Dapretto, 2006) and is in accord with a larger body of less direct evidence that has shown that the BOLD signal in fMRI studies and the $\mu$-power in EEG studies have similar properties. Both $\mu$-suppression and fMRI signals show a somatotopic organization that allows discriminating actions performed by different effectors (Pfurtscheller et al., 1997; Gazzola et al., 2006; Etzel et al., 2008). Both respond more to goal-directed transitive actions than meaningless intransitive actions (Buccino et al., 2001; Muthukumaraswamy et al., 2004). Both show higher signals when the observer has expertise in the particular action (Calvo-Merino et al., 2005; Orgs et al., 2008). Finally, both show predictive signals before an action that can be anticipated (Caetano et al., 2007; Southgate et al., 2009; Thioux et al., 2009).

In summary, with mirror neurons first discovered in the ventral premotor cortex (Gallese et al., 1996; Umiltà et al., 2001; Kohler et al., 2002; Keysers et al., 2003), many assumed that $\mu$-suppression quantifies activity in mirror neurons in general and in BA44 in particular (Pineda, 2005). Our results support the idea that $\mu$-suppression measures activity of regions associated with the MNS, but they argue against the notion that $\mu$-suppression primarily measures mirror activity in BA44: BOLD activity in BA2, the IPL and the APM robustly and significantly correlated with $\mu$-suppression during action observation and execution but that in BA44 did not. Additionally, our data suggest that although EEG and fMRI tasks have used somewhat different motor tasks in the past to test activity in the MNS, they have actually measured activity in overlapping neural substrates. We therefore hope that our findings provide a basis for integrating more closely the burgeoning but often separate literatures on the MNS using fMRI and EEG. By suggesting that $\mu$-suppression may correlate more with BA2, IPL and DPM, than BA44 activity, we hope that our results will shed further light on the sometimes apparently contrasting findings in the study of patients with impairments of social cognition (Oberman et al., 2005; Dinstein et al., 2010).

\section{Notes}

Supplemental material for this article is available at http://www.nin.knaw.nl/ Portals/0/Department/keysers/Arnstein\%20SupplementaryFigures.pdf. For more information about EPI artifacts correction, scalp topography of mu-power, and unsmoothed data analysis, you can visit the supplementary file. This material has not been peer reviewed.

\section{References}

Allen PJ, Polizzi G, Krakow K, Fish DR, Lemieux L (1998) Identification of EEG events in the MR scanner: the problem of pulse artifact and a method for its subtraction. Neuroimage 8:229-239.

Allen PJ, Josephs O, Turner R (2000) A method for removing imaging artifact from continuous EEG recorded during functional MRI. Neuroimage 12:230-239.

Buccino G, Binkofski F, Fink GR, Fadiga L, Fogassi L, Gallese V, Seitz RJ, Zilles K, Rizzolatti G, Freund HJ (2001) Action observation activates premotor and parietal areas in a somatotopic manner: an fMRI study. Eur J Neurosci 13:400-404.

Caetano G, Jousmäki V, Hari R (2007) Actor's and observer's primary motor cortices stabilize similarly after seen or heard motor actions. Proc Natl Acad Sci U S A 104:9058-9062.

Calvo-Merino B, Glaser DE, Grèzes J, Passingham RE, Haggard P (2005) Action observation and acquired motor skills: an FMRI study with expert dancers. Cereb Cortex 15:1243-1249.

Caspers S, Zilles K, Laird AR, Eickhoff SB (2010) ALE meta-analysis of action observation and imitation in the human brain. Neuroimage 50:1148-1167.

Cisek P, Kalaska JF (2004) Neural correlates of mental rehearsal in dorsal premotor cortex. Nature 431:993-996.

Dinstein I, Hasson U, Rubin N, Heeger DJ (2007) Brain areas selective for both observed and executed movements. J Neurophysiol 98:1415-1427.

Dinstein I, Thomas C, Humphreys K, Minshew N, Behrmann M, Heeger DJ (2010) Normal movement selectivity in autism. Neuron 66:461-469.

Etzel JA, Gazzola V, Keysers C (2008) Testing simulation theory with crossmodal multivariate classification of fMRI data. PLoS One 3:e3690.

Evangeliou MN, Raos V, Galletti C, Savaki HE (2009) Functional imaging of the parietal cortex during action execution and observation. Cereb Cortex 19:624-639.

Filimon F, Nelson JD, Hagler DJ, Sereno MI (2007) Human cortical representations for reaching: mirror neurons for execution, observation, and imagery. Neuroimage 37:1315-1328.

Gallese V, Fadiga L, Fogassi L, Rizzolatti G (1996) Action recognition in the premotor cortex. Brain 119:593-609.

Gastaut H (1952) Etude électrocorticographique de la réactivité des rythmes rolandiques. Rev Neurol 87:176-182.

Gazzola V, Keysers C (2009) The observation and execution of actions share motor and somatosensory voxels in all tested subjects: single-subject analyses of unsmoothed fMRI data. Cereb Cortex 19:1239-1255.

Gazzola V, Aziz-Zadeh L, Keysers C (2006) Empathy and the somatotopic auditory mirror system in humans. Curr Biol 16:1824-1829.

Gazzola V, Rizzolatti G, Wicker B, Keysers C (2007) The anthropomorphic brain: the mirror neuron system responds to human and robotic actions. Neuroimage 35:1674-1684.

Grèzes J, Armony JL, Rowe J, Passingham RE (2003) Activations related to "mirror" and "canonical" neurones in the human brain: an fMRI study. Neuroimage 18:928-937.

Hari R, Forss N, Avikainen S, Kirveskari E, Salenius S, Rizzolatti G (1998) Activation of human primary motor cortex during action observation: a neuromagnetic study. Proc Natl Acad Sci U S A 95:15061-15065.

Harmon-Jones E (2006) Unilateral right-hand contractions cause contralateral alpha power suppression and approach motivational affective experience. Psychophysiology 43:598-603. 
Iacoboni M, Dapretto M (2006) The mirror neuron system and the consequences of its dysfunction. Nat Rev Neurosci 7:942-951.

Keysers C (2009) Mirror neurons. Curr Biol 19:R971-R973.

Keysers C, Gazzola V (2009) Expanding the mirror: vicarious activity for actions, emotions, and sensations. Curr Opin Neurobiol 19:666-671.

Keysers C, Kohler E, Umiltà MA, Nanetti L, Fogassi L, Gallese V (2003) Audiovisual mirror neurons and action recognition. Exp Brain Res 153:628-636.

Keysers C, Kaas JH, Gazzola V (2010) Somatosensation in social perception. Nat Rev Neurosci 11:417-428.

Kilner JM, Neal A, Weiskopf N, Friston KJ, Frith CD (2009) Evidence of mirror neurons in human inferior frontal gyrus. J Neurosci 29:10153-10159.

Kohler E, Keysers C, Umiltà MA, Fogassi L, Gallese V, Rizzolatti G (2002) Hearing sounds, understanding actions: action representation in mirror neurons. Science 297:846-848.

Mukamel R, Ekstrom AD, Kaplan J, Iacoboni M, Fried I (2010) Singleneuron responses in humans during execution and observation of actions. Curr Biol 20:750-756.

Muthukumaraswamy SD, Johnson BW, McNair NA (2004) Mu rhythm modulation during observation of an object-directed grasp. Brain Res Cogn Brain Res 19:195-201.

Oberman LM, Hubbard EM, McCleery JP, Altschuler EL, Ramachandran VS, Pineda JA (2005) EEG evidence for mirror neuron dysfunction in autism spectrum disorders. Brain Res Cogn Brain Res 24:190-198.

Ohara S, Ikeda A, Kunieda T, Yazawa S, Baba K, Nagamine T, Taki W, Hashimoto N, Mihara T, Shibasaki H (2000) Movement-related change of electrocorticographic activity in human supplementary motor area proper. Brain 123:1203-1215.

Orgs G, Dombrowski JH, Heil M, Jansen-Osmann P (2008) Expertise in dance modulates alpha/beta event-related desynchronization during action observation. Eur J Neurosci 27:3380-3384.

Perry A, Bentin S (2009) Mirror activity in the human brain while observing hand movements: a comparison between EEG desynchronization in the mu-range and previous fMRI results. Brain Res 1282:126-132.

Pfurtscheller G, Neuper C, Andrew C, Edlinger G (1997) Foot and hand area mu rhythms. Int J Psychophysiol 26:121-135.

Pineda JA (2005) The functional significance of mu rhythms: translating "seeing" and "hearing" into "doing." Brain Res Brain Res Rev 50:57-68.

Pineda JA, Allison BZ, Vankov A (2000) The effects of self-movement, ob- servation, and imagination on mu rhythms and readiness potentials (RP's): toward a brain-computer interface (BCI). IEEE Trans Rehabil Eng 8:219-222.

Raos V, Evangeliou MN, Savaki HE (2004) Observation of action: grasping with the mind's hand. Neuroimage 23:193-201.

Raymaekers R, Wiersema JR, Roeyers H (2009) EEG study of the mirror neuron system in children with high functioning autism. Brain Res 1304:113-121.

Rizzolatti G, Sinigaglia C (2010) The functional role of the parieto-frontal mirror circuit: interpretations and misinterpretations. Nat Rev Neurosci 11:264-274.

Rizzolatti G, Fabbri-Destro M, Cattaneo L (2009) Mirror neurons and their clinical relevance. Nat Clin Pract Neurol 5:24-34.

Rozzi S, Ferrari PF, Bonini L, Rizzolatti G, Fogassi L (2008) Functional organization of inferior parietal lobule convexity in the macaque monkey: electrophysiological characterization of motor, sensory and mirror responses and their correlation with cytoarchitectonic areas. Eur J Neurosci 28:1569-1588.

Salmelin R, Hari R (1994) Spatiotemporal characteristics of sensorimotor neuromagnetic rhythms related to thumb movement. Neuroscience 60:537-550.

Salmelin R, Hämäläinen M, Kajola M, Hari R (1995) Functional segregation of movement-related rhythmic activity in the human brain. Neuroimage 2:237-243.

Southgate V, Johnson MH, Osborne T, Csibra G (2009) Predictive motor activation during action observation in human infants. Biol Lett $5: 769-772$

Thioux M, Greven I, Gazzola V, Keysers C (2009) Assessing hand actions with the mirror neuron system. In: 15th Annual Meeting of the Organization for Human Brain Mapping. San Francisco, CA, June.

Turella L, Erb M, Grodd W, Castiello U (2009) Visual features of an observed agent do not modulate human brain activity during action observation. Neuroimage 46:844-853.

Umiltà MA, Kohler E, Gallese V, Fogassi L, Fadiga L, Keysers C, Rizzolatti G (2001) I know what you are doing. a neurophysiological study. Neuron 31:155-165.

Ziehe A, Müller KR, Nolte G, Mackert BM, Curio G (2000) Artifact reduction in magnetoneurography based on time-delayed second-order correlations. IEEE Trans Biomed Eng 47:75-87. 